\title{
Pathology of intrahepatic cholangiocarcinoma
}

\author{
Sandrine Vijgen ${ }^{1}$, Benoit Terris ${ }^{2}$, Laura Rubbia-Brandt ${ }^{1}$ \\ ${ }^{1}$ Division of Clinical Pathology, Geneva University Hospital and Faculty of Medicine of Geneva, Geneva, Switzerland; ${ }^{2}$ Department of \\ Histopathology, Paris Descartes University, Assistance Publique-Hôpitaux de Paris, Cochin University Hospital, Paris, France \\ Contributions: (I) Conception and design: All authors; (II) Administrative support: All authors; (III) Provision of study material or patients: All authors; \\ (IV) Collection and assembly of data: All authors; (V) Data analysis and interpretation: All authors; (VI) Manuscript writing: All authors; (VII) Final \\ approval of manuscript: All authors. \\ Correspondence to: Professor Laura Rubbia-Brandt, MD, PhD. Division of Clinical Pathology, Geneva University Hospital and Faculty of Medicine of \\ Geneva, Geneva, Switzerland. Email: laura.rubbia-brandt@hcuge.ch.
}

\begin{abstract}
Intrahepatic cholangiocarcinoma (iCC) is a primary carcinoma of the liver with increasing significance and major pathogenic, clinical and therapeutic challenges. Classically, it arises from malignant transformation of cholangiocytes bordering small portal bile duct (BD) to second-order segmental large BDs. It has three major macroscopic growth pattern [mass-forming (MF), periductal infiltrative (PI), and intraductal growth (IG)] and histologically is a desmoplastic stroma-rich adenocarcinoma with cholangiocyte differentiation. Recent data pointed out noteworthy degree of heterogeneity in regards of their epidemiology and risk factors, pathological and molecular features, pathogenesis, clinical behaviors and treatment. Notably, several histological variants are described and can coexist within the same tumor. Several different cells of origin have also been depicted in a fraction of iCCs, amongst which malignant transformation of ductules, of hepatic stem/progenitor cells, of periductal glands or through oncogenic reprogramming of adult hepatocytes. A degree of pathological overlap with hepatocellular carcinoma (HCC) may be observed in a portion of iCC. A series of precursor lesions are today characterized and emphasize the existence of a multistep carcinogenesis process. Overall, these new data have brought up in proposal of new histological or molecular classifications, which could soon replace current anatomic-based classification and could have major impact on establishment of prognosis and on development of novel target treatment approaches.
\end{abstract}

Keywords: Intrahepatic cholangiocarcinoma (iCC); macroscopic features; microscopic variants; immunohistochemistry; cells of origin

Submitted Jun 20, 2016. Accepted for publication Oct 09, 2016.

doi: $10.21037 / \mathrm{hbsn} .2016 .11 .04$

View this article at: http://dx.doi.org/10.21037/hbsn.2016.11.04

\section{Introduction}

Primary hepatic malignant epithelial neoplasia includes several different types of tumors, amongst which cholangiocarcinoma (CC), $2^{\text {nd }}$ most frequent cancer of the liver after hepatocellular carcinoma (HCC). CC is currently classified according to the World Health Organization (WHO) (1) and Union for International Cancer Control (UICC) (2) into two major categories, in reference to the anatomic origin along the biliary tract: (I) intrahepatic cholangiocarcinoma (iCC), also assigned as peripheral CC (term that tends to be discouraged today) and representing approximately $20 \%$ of CC; iCC develops within liver, topographically from the second-order bile duct (BD) (segmental) and more proximal intrahepatic BD (septal, interlobular BD and ductule); (II) extrahepatic bile duct carcinoma (eBDC), accounting for $80 \%$ of CC; eBDC includes tumors arising from large hepatic hilar BD (also called Klatskin tumor) to more distal extrahepatic BDs excluding those arising from Vater's ampulla. As a tumor mass, at time of diagnosis, can extend from the hilum to intrahepatic perihilar parenchyma, the determination of its 

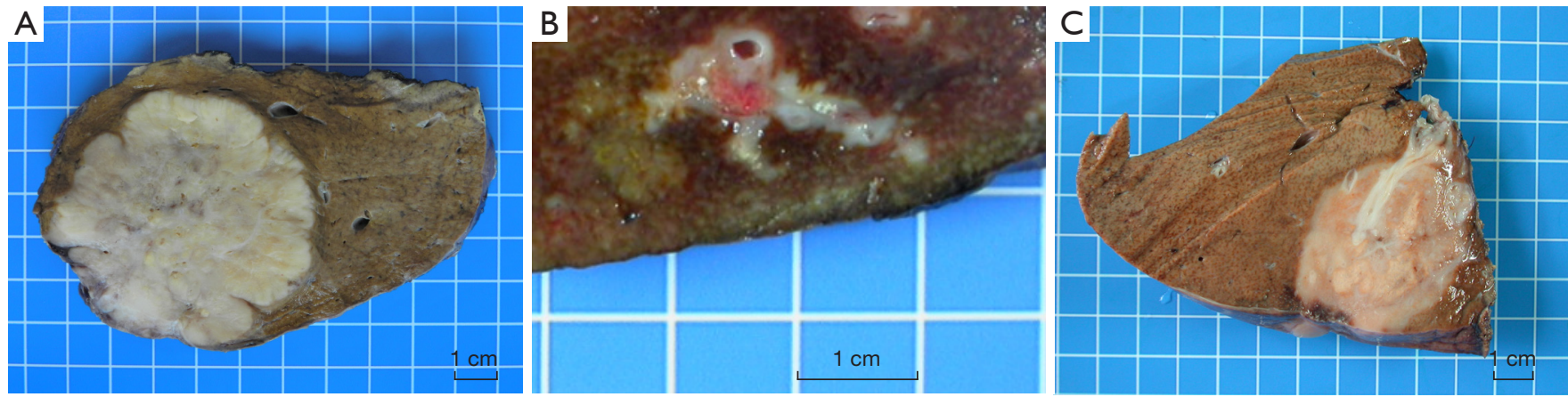

Figure 1 Macroscopic patterns of intrahepatic cholangiocarcinoma (iCC). (A) Mass-forming type consisting of a single solid and lobulated mass with no connection macroscopically discernible with a bile duct. It is characterized by irregular but well-defined and not encapsulated borders; (B) periductal infiltrating type characterized by growth along a bile duct, without mass formation; (C) mixed pattern associating mass-forming type and periductal infiltrating type.

anatomic origin either as intrahepatic or extrahepatic CC may be difficult, or even impossible. Today's consensus is to classify Klatskin tumor as a form of eBDC separately from the more distal eBDC (3). This current anatomical-based classification of $\mathrm{CC}$ rises some problem notably in accurate assessment of epidemiological background, carcinogenesis and patients' outcome $(4,5)$.

Several other classifications are now emerging based on extent of the disease, gross morphology, histopathology, tumor biology or postulated cell of origin. Although they all provide helpful information, they are not totally yet welldefined or accurate. Therefore, despite important increases in knowledge, further studies are needed to additionally improve iCC understanding and its subclassification (6).

\section{Pathology of iCC}

iCC is most commonly an adenocarcinoma with biliary differentiation arising in any segment of the intrahepatic biliary tree, from the peripheral periportal ductules and small portal BDs to the perihilar segmental ducts; iCC may also arise from intrahepatic peribiliary glands (PBGs), hepatic stem/progenitor cells (HPCs) and other cells (see below).

\section{Macroscopic pattern}

According to its macroscopic appearance, the Liver Cancer Study Group of Japan (7-11) has subdivided iCC into four categories: (I) the mass-forming (MF) type, consisting of a grey-white, well-delimited, firm and solid, non-encapsulated, polylobulated mass within the liver at distance from the hilum and with no connection macroscopically discernible with a BD (Figure 1A); (II) the periductal infiltrating type (PI), characterized by a tumoral growth pattern spreading along intrahepatic portal tracts (Figure 1B), together with stenosis of the involved ducts and upstream BDs obstructive dilatation and cholangitis; (III) the intraductal growth (IG) type, typified by a polypoid or papillary tumor mass growing within the lumen of a dilated large $\mathrm{BD}$; (IV) the mixed pattern (Figure 1C).

MF type is the most prevalent, accounting for about $65 \%$ of all iCCs, while PI and IG type are much less common, representing in $6 \%$ and $4 \%$ of all iCCs, respectively $(10,12)$. Mixed pattern (around 25\% of iCC) combines mostly MF and PI types. iCC deriving from malignant transformation of ductules and small portal BDs usually ends preferentially in a MF type, whereas iCC arising within large secondorder or segmental intrahepatic BDs usually results in PI, IG or mixed type. iCC may be associated with non-invasive intraductal papillary neoplasm of the bile duct (IPNB) (see below), which extends intraductally and superficially along the surrounding $\mathrm{BD}$ epithelium.

At advanced stages, intrahepatic metastases occur consisting of various sized nodules, which may coalesce; regional lymph nodes and lung metastases may arise (13). In addition, at this stage, anatomical classification has some limitations: $\mathrm{iCC}$ of PI or IG type associated with an invasive mass (MF) or MF involved by cancerization of a BD (PI or IG), complicating the determination of the initial type of iCC.

Clinical features and biological behaviors of iCC differ among the different macroscopic types (14). MF-type without hilar invasion have after surgery a lower outcome 

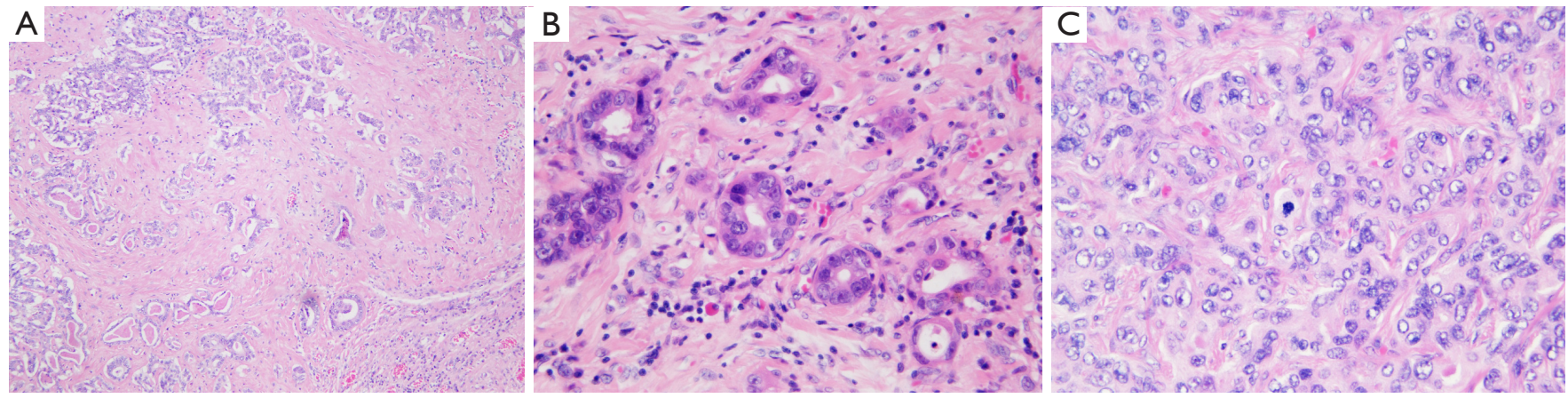

Figure 2 Traditional intrahepatic cholangiocarcinoma (iCC). (A) Hematoxylin and eosin stain. ICCs typically elicit prominent fibrodesmoplastic stromal reaction (original magnification, $\times 10$ ); (B) hematoxylin and eosin stain. Moderately differentiated iCC characterized by infiltrative irregular glands lined by columnar-shaped and moderately pleomorphic cells with eosinophilic cytoplasm (original magnification, $\times 40$ ); (C) hematoxylin and eosin stain. Poorly differentiated iCC displaying a solid growth pattern, without identifiable glandular lumens and composed of more highly atypical cells (original magnification, $\times 40$ ).

than PI-type with 5-year survival rates of $41.2 \%$ and $85.7 \%$, respectively $(\mathrm{P}=0.032)$. PI- or IG-type may be associated with jaundice, intrahepatic biliary fibrosis and cholangitis. Mixed MF- and PI-types is mostly coupled with portal vein invasion, lymph node involvement and positive surgical margins, and patients have often a more unfavorable prognosis, even after complete surgical resection, than those with other types of iCC (14). Lymph node invasion rate of the IG-type is lower than those of the other types (IG: $0 \%$; MF: $16 \%$; MF + PI: $50 \%$; and PI: 66\%).

\section{Histological pattern}

Common histological feature of iCC is well-to-moderately differentiated adenocarcinoma with varying degrees of desmoplasia; often, the abundant stroma is variably distributed (Figure 2A). Tumor center is habitually more densely fibrotic (intermingled only with sparse tumoral cells, combined to occasionally focal calcifications or necrosis), while the periphery has more abundant and proliferating tumoral cells, that infiltrate the surrounding parenchyma either by compression, infiltration along the sinusoids or replacement of hepatocytes in their cords. Portal tracts are coopted within the mass. Portal venules, lymphatic vessels and intrahepatic nerves are often invaded, already at an early stage.

Traditionally iCC is subclassified in classic adenocarcinoma (Figure 2) and rare histological variants such as adenosquamous and squamous carcinoma, mucinous carcinoma (often with mucin visible at cut surface and intraductal-growth pattern, occasionally associated with intestinal type goblet cells), signet-ring cell carcinoma, clear cell carcinoma (with abundant clear cytoplasm), lymphoepithelioma-like carcinoma, neuroendocrine type or may have sarcomatous area, mimicking a spindle cell sarcoma (Figure 3).

However based on latest research data, new histological classifications of iCC have been proposed (4) that are summarized in Table 1.

One classification subcategorized iCC in mucin-iCC versus mixed-non mucin iCC (15). Mucin-iCC develops from columnar mucin-producing cholangiocytes that line large intrahepatic BD (segmental and septal BD) and is characterized by mucin-producing CC areas intermingled with abundant dense and hyalinized fibrous stroma. MixediCC develops from mucin-negative cuboidal cholangiocytes lining small intra-hepatic BD (interlobular BD and ductules). Ductules harbor HPCs which can differentiate into both hepatocytes and cholangiocytes and could give rise to iCC (see below). Clinicopathologically, both muciniCCs and hilar eCCs are characterized by a predominantly hilar location, smaller tumor size, single tumor and more lymphatic and perineural invasion compared with mixed-iCCs and cholangiocarcinoma (CLCs) (see below) predominantly with peripheral location, occasionally multiple tumors, larger tumor size, less lymphatic and perineural invasion.

Another classification subdivided iCC in conventional versus unconventional subtype (12). Conventional iCC commonly occurs in liver without underlying disease (>85\%) and corresponds to a classic adenocarcinoma with biliary features. Unconventional iCC is matter of major research especially on its histogenesis and pathogenesis; by contrast to 

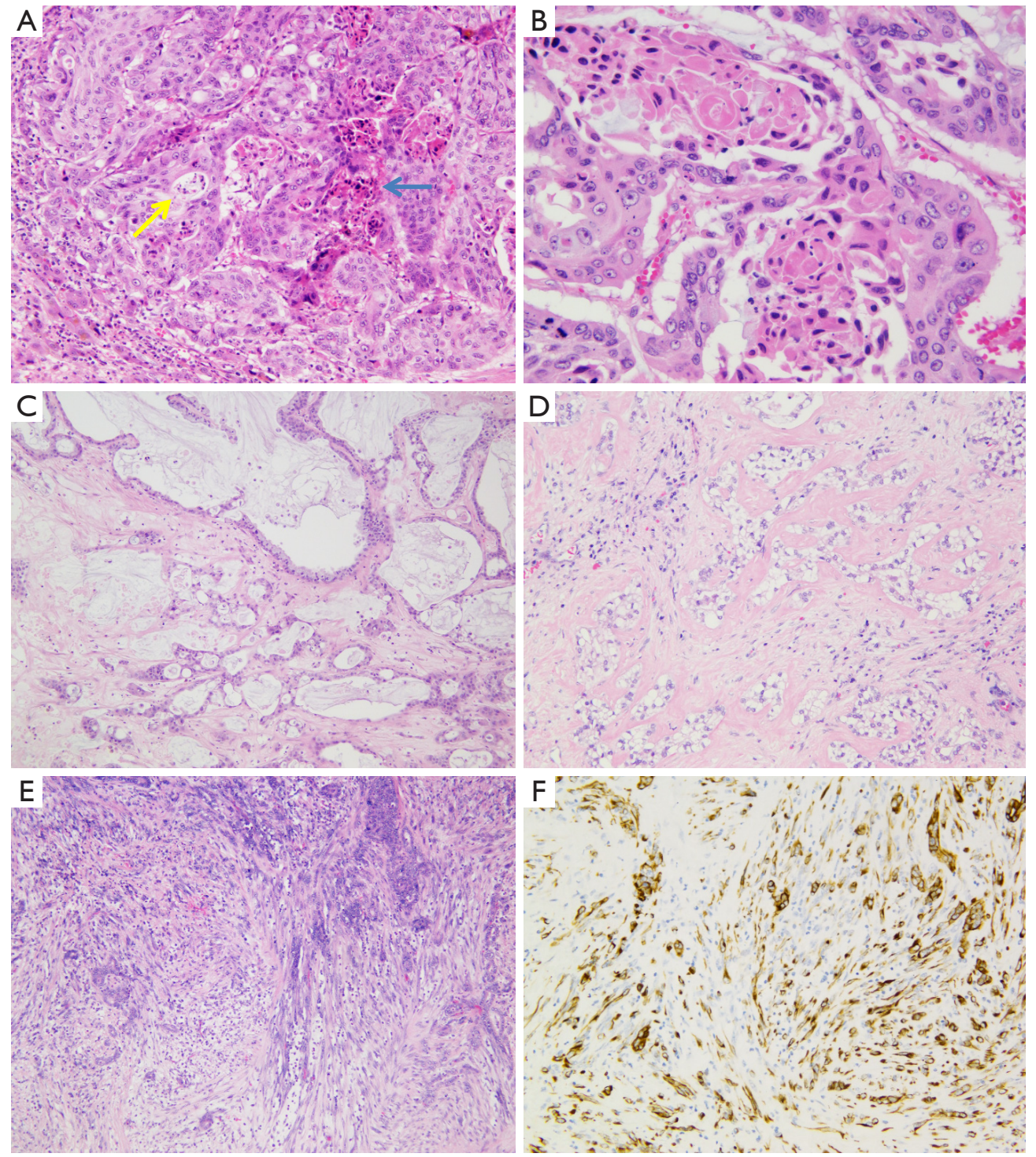

Figure 3 Rare histological variants of intrahepatic cholangiocarcinoma (iCC). (A) Hematoxylin and eosin stain. Adenosquamous variant of iCC showing two components: a squamous component (blue arrow) and a glandular and trabecular component (yellow arrow) (original magnification, $\times 20$ ); (B) hematoxylin and eosin stain. Dyskeratotis is seen within groups of tumoral cells in the squamous component (original magnification, $\times 40$ ); (C) hematoxylin and eosin stain. Mucinous variant of traditional iCC containing large tumoral glands lined by a mucinous neoplastic epithelium with luminal mucin accumulation (original magnification, $\times 10$ ); (D) hematoxylin and eosin stain. Clear cell type of iCC characterized by cells showing an abundant clear cytoplasm (original magnification, $\times 20$ ); (E) hematoxylin and eosin stain. Sarcomatoid variant of iCC showing large sheets of spindled cells intermingled with few glandular structures (original magnification, $\times 10$ ); (F) pan-cytokeratin stain. The spindled cells are keratin positive, confirming their carcinomatous nature (original magnification, $\times 20$ ).

conventional iCCs, it most often develops on a background of a non-biliary chronic liver disease and cirrhosis (>55\%) and displays five different histological variants (both at architectural and cytological levels): (I) trabecular subtype, consisting of polygonal eosinophilic tumoral cells organized in thick, occasionally anastomosing trabeculae, mimicking HCC. It however varies from HCC by the presence, in tumoral mass, of central fibrosis intermingled with sparse tumoral cells, presence of calcifications and cytologically tumoral cells with indistinct nucleoli, no bile production 
Table 1 Traditional and new classifications of intrahepatic cholangiocarcinoma

Traditional classification Ne
classification of iCC of iCC
Adenocarcinoma
Well differentiated
Moderately differentiated
Poorly differentiated
Rare variants
Adenosquamous type
Squamous type
Mucinous type
Signet-ring cell type
Clear cell type
Lymphoepithelial type
Sarcomatoid type
Others

New classification of iCC by Komuta et al. (15)

Mucin-iCC (large bile duct type)

Mixed-iCC (small bile duct type)

Cholangiolocellular carcinoma

New classification of iCC by Sempoux et al. (12)

Conventional iCC (with biliary features)

Unconventional iCC

Trabecular subtype

Hilar subtype

Intraductal neoplasia of intrahepatic bile ducts

IPNB

ITNB

iCC with predominant (ductal plate malformation)

Cholangiolocellular carcinoma

iCC, intrahepatic cholangiocarcinoma; IPNB, intraductal papillary neoplasm of the bile duct; ITNB, intraductal tubulopapillary neoplasm.

and distinct immunohistochemical characteristics; (II) hilar subtype mimicking typical hilar eCC. This iCC variant displays cords, tubular or large irregular dilated tumoral glands with clear atypical features, hyperchromatic nuclei and mucin production. It shows extensive periductal or papillary intraductal invasion similar to conventional iCC, perineural invasion, and PBGs are often invaded. It most likely corresponds to an iCC originating from large $\mathrm{BDs}$ that have progressed into MF iCC, to a PBG carcinoma or to a conventional iCC with secondary involvement of intrahepatic large BDs. Intraductal dysplasia may be present; (III) intraductal neoplasia of intrahepatic BD often characterized by a superficial spreading pattern and with two major variants: (i) intraductal papillary neoplasm of $\mathrm{BD}$, ranging from preneoplastic IPNB (Figure 4A) (see below) to well differentiated papillary invasive frequently mucinous adenocarcinoma. They characteristically correspond macroscopically to the IG type; (ii) intraductal tubular neoplasm of bile duct (ITNB), rare and mainly characterized by tubular glands, rarely papillary, devoid of mucin, that cast and obstruct the dilated biliary duct; (IV) iCC with predominant "ductal plate malformation" pattern showing irregularly dilated neoplastic glands associated to an important desmoplastic fibrosis $(16,17)$ (Figure 4B,C); (V) cholangiolocellular carcinoma (CLC) (Figure 5) (see below).

\section{Immunohistochemistry}

iCC may further be characterized by immunohistochemistry. Tumor cells are positive for biliary subtype of cytokeratins, namely cytokeratins 7 and 19 but no specific markers still exist. iCC heterogeneity may be underlined by immunohistochemical expression and gene expression profiling $(4,12,15)$. Notably, $\mathrm{N}$-cadherin expression is significantly increased in iCC compared with eCC, with a specificity for the diagnosis of $\mathrm{iCC}$ of $88 \%$ that may reaches 98\% if combined with CK7. Mucin-iCC shows cytoplasmic/ nuclear positivity for protein S100, MUC1 positivity, intense cytoplasmic positivity for EMA and EpCAM but no positivity for NCAM (CD56) $(15,18,19)$. Ductular areas in mixed-iCCs and CLCs show similar immunohistochemical profiles to ductules/HPCs; they demonstrate membranous positivity for NCAM (CD56), cytoplasmic positivity for ANNX3, EpCAM, and TACSTD2, and apical positivity for EMA. Protein S100 is negative in both the ductular areas of mixed-iCCs and CLCs and ductules/HPCs.

Hepatocytic markers are observed occasionally in iCCs that are otherwise devoid of hepatocyte morphology, such as HepPar1 and arginase 1 (20). Albumin mRNA in situ hybridization (ISH) is a sensitive and highly specific diagnostic tool for distinguishing iCC, in particular mixed- 

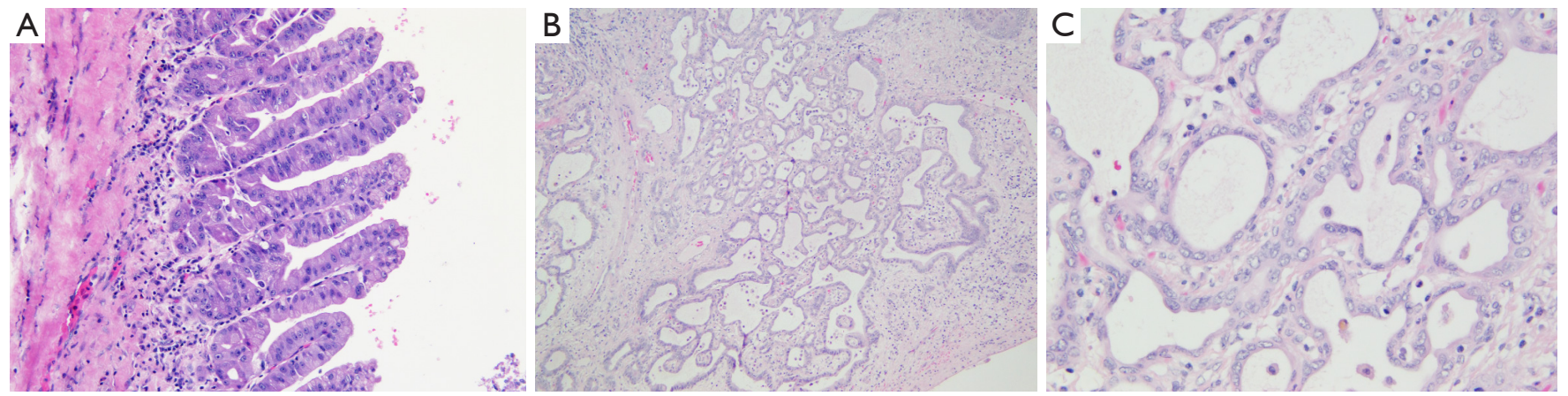

Figure 4 Unconventional intrahepatic cholangiocarcinoma (iCC). (A) Hematoxylin and eosin stain. Intraductal papillary neoplasm of the bile duct (IPNB) consisting of papillary proliferation in the lumen of a dilated large bile duct. Papillae are characterized by a delicate fibrovascular axis and are lined by atypical columnar cells (original magnification, $\times 20$ ); (B) hematoxylin and eosin stain. ICC with predominant ductal plate malformation corresponding to a vaguely nodular carcinomatous area composed of irregularly dilated neoplastic glands (original magnification, $\times 10$ ); (C) hematoxylin and eosin stain. The dilated lumens of these tumoral glands are lined by a single layer of cuboidal epithelial cells (original magnification, $\times 40$ ).
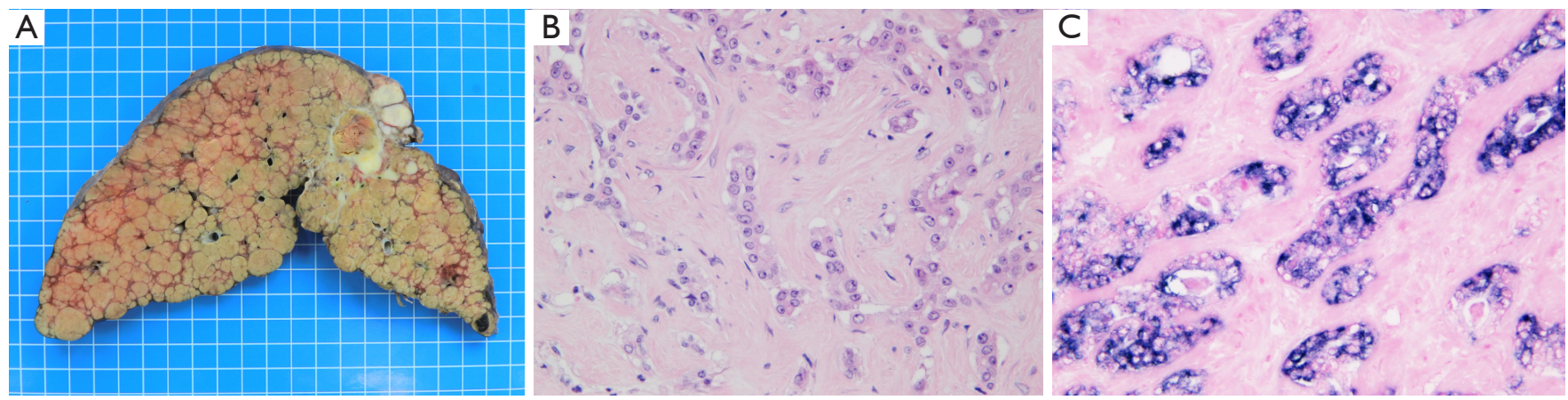

Figure 5 Cholangiolocellular carcinoma (CLC). (A) Gross specimen of a 38 years old patient who was transplanted for a CLC developed in a cirrhotic liver due to chronic hepatitis B virus infection. Grossly, this lesion corresponds to a mass-forming type with central yellowish necrotic area and several satellite nodules; (B) hematoxylin and eosin stain. CLC consists of well differentiated ductular glands lined with mild atypical cells and growing in a tubular, cord-like and anastomosing pattern (original magnification, $\times 40)$; (C) albumin mRNA in situ hybridization (ISH). Most of the tumor cells demonstrate a cytoplasmic hybridization signal (original magnification, $\times 40$ ).

iCCs and CLCs (positive) from perihilar adenocarcinoma but also from metastatic adenocarcinoma, particularly pancreatic ductal adenocarcinoma (negative) $(15,21)$.

\section{Precursor lesions of icc}

It is now recognized that iCC proceeds through multistepcarcinogenesis; three precursor lesions have been at present morphologically established (22-31): (I) a flat intraepithelial bilirary neoplasia or BillN (discernible only at level of microscope) (Figure 6A); (II) an IPNB (previously named papillomatosis) (discernible on radiologic imaging or at macroscopic examination (Figures $4 A$ and $6 B$ ); and (III) intraductal tubulo-papillary neoplasm (ITNB). These lesions are generally observed in large intrahepatic, hilar and extrahepatic BDs, and are infrequently observed in the septal or interlobular BDs. BillN may be seen in peri-biliary glands.

Both take place usually in the context of chronic inflammatory biliary diseases, such as hepatolithiasis, primary sclerosing cholangitis, infestation by liver flukes, as well as congenital biliary diseases. BillN is also observed in chronic $\mathrm{HCV}$ hepatic disease. BillN is characterized by a proliferation of cholangiocytes with different levels of nuclear atypia (with increased nuclei/cytoplasmic ratio, nuclear hyperchromasia and loose of cell polarity) and 

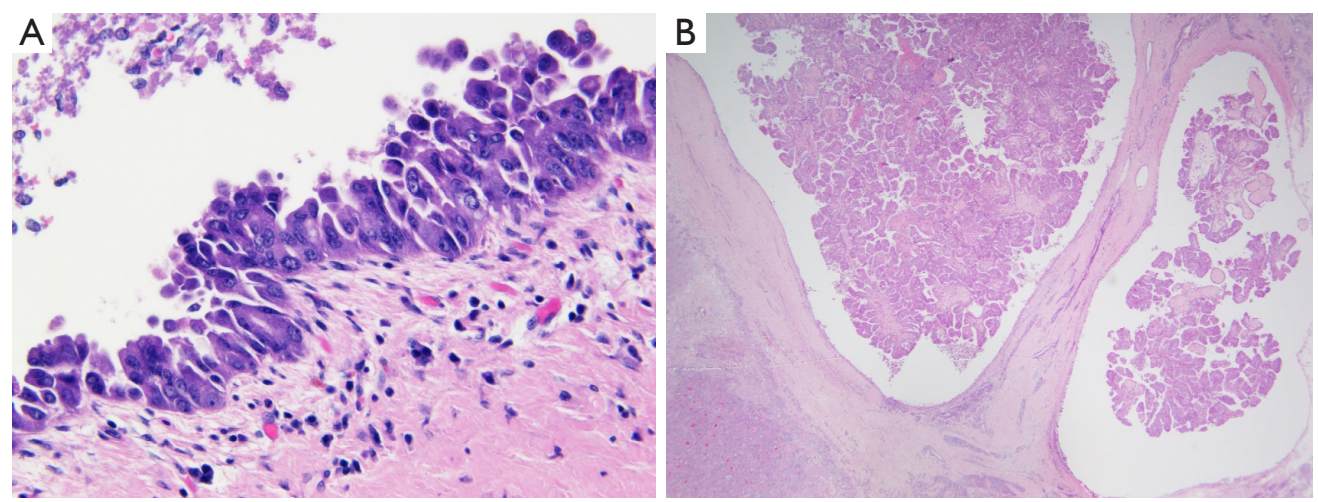

Figure 6 Precursor lesions of intrahepatic cholangiocarcinoma (iCC). (A) Hematoxylin and eosin stain. High grade biliary intraepithelial neoplasia (BillN) characterized by atypical epithelial cells with multilayering of nuclei (original magnification, $\times 40$ ); (B) hematoxylin and eosin stain. Intraductal papillary neoplasm of the bile duct (IPNB) of a large bile duct which was discernible at macroscopic level (original magnification, $\times 2$ ).

plusistratified, flat or micropapillary epithelium. It is substratified in BillN-1 and -2 (low-grade) and BillN-3 (high grade). These lesions are commonly observed in the intrahepatic large BD, PBGs and extrahepatic hilar and distal BDs. BillN demonstrates a step-wise expression of S100P and oncogene-related gene, as p21, cyclin D1 and p53 and a decrease of SMAD4. They characteristically give raise to conventional invasive iCC (tubular adenocarcinoma), whereas IPNB may progress to either colloid carcinoma (mucinous carcinoma) or conventional iCC.

IPNB are more and more admitted as the biliary counterpart of intraductal papillary mucinous neoplasm (IPMN) of the pancreas, while intraductal tubular neoplasm of bile duct (ITNB) is considered as the biliary counterpart of intraductal tubular neoplasm of the pancreas (23). Both biliary and pancreatic duct derive embryonically from the foregut. IPNB is characterized by papillary biliary proliferation that may be $>10 \mathrm{~mm}$ (4). Papillae have a delicate fibrovascular axis and are lined by an epithelium that may have four different phenotypes: intestinal, gastric, pancreatobiliary and oncocytic. These lesions may show in one-third of cases an excessive mucosecretion within the lumen of the biliary duct. Based on the degree of atypia of the epithelium, they may be divided in low and high grade of dysplasia. ITNB is another polypoid lesion with a predominant tubular growth pattern. The tubular glands are densely packed back-to-back with occasional papillary elements or solid areas, growing within BD lumens that are bordered by cuboidal to columnar mildly atypical cells. ITNB sometimes contains some necrotic areas, with a comedocarcinoma-like pattern.

\section{Recent data about cells of origin if iCCs}

Recent research has brought up several new insights into the cellular origin of iCC (referring to a normal cell that acquires the first cancer-initiating mutation), indicative of possible multiple cell lineages origin $(32,33)$. Histogenesis complexity and different molecular mechanisms underlying the diverse growth patterns of this malignancy is an object of clinical concern. In this regard, iCC classification based on cellular origin could take increasingly importance supported by emerging data revealing differences in pathological, radiological and clinical findings. This type of classification could be more in accordance with present knowledge on epidemiology and risk factors.

It is today considered that iCC can arise from any type of liver cell due to the cellular plasticity that might be caused by reprogramming mechanisms. iCC may develop from intrahepatic epithelial lining of small to large BD and PBGs $(32,33)$, from malignant transformation of ductules, from HPCs of canal of Hering or periductal glands stem niche or through oncogenic reprogramming of adult hepatocytes. However, these conclusions are largely based on reliance on results from murine models of lineage tracing systems which have some known technical limitations.

In human, it is today considered that iCC arises from topographically heterogeneous cholangiocytes within the different levels of the biliary tree (32,34-36). Cylindrical mucin-producing cholangiocytes border large BDs while cuboidal non-mucin-producing cholangiocytes compose ductules, where bipotential hepatic progenitor cells (HPCs) are also located (stem cell niche). MF type of iCC most 
likely arises from interlobular BD, ductules and HPCs, located in the canal of Hering. Large BD mucin-iCCs have equivalent clinico-pathological, immunohistochemical, and gene expression profiles to hilar eCCs, and similar profile to cylindrical, taller, mucin-producing cholangiocytes that line hilar and intrahepatic large BDs. In contrast, mixed-iCCs (small BD type) share similarities with CLCs and CK19positive HCCs.

Until recently, HCC and iCC were considered as distinct clinical and pathological entities with different pathogenesis. HCC commonly arises on a setting of advanced chronic liver diseases as cirrhosis, while iCC mainly arises on the background of a normal liver. HCC and iCC were assume deriving through dedifferentiation from mature hepatocytes and biliary cells (cholangiocytes), respectively. While this remains generally correct, recent insights in pathogenesis of combined hepato-cholangiocarcinoma (a mixed malignant hepatic tumor of the adult, mixed HCC-CC) underlined the possibility of histological and phenotypic overlap between HCC and iCC (see beelow).

\section{Recent data about cholangiolocellular carcinomas}

CLC is characterized by a proliferation of very regular and well differentiated ductular glands bordered by only very mildly atypical cells and surrounded by fibrosis, mimicking a ductal plate malformation (Figure 5) (16,37).

A number of observations suggest that CLCs, together with bile ductular (mixed)-type iCCs and CK19+ HCCs represent a group of primitive liver cancers originating from HPCs, the different phenotype depending on the step of hepatic progenitor cell differentiation toward cholangiocytes or hepatocytes, in which neoplastic transformation occurs $(4,38)$.

Classification of this variant of primary liver cancer is a matter of debate and lack of complete consensus (38). It is, by certain authors, categorized into a subtype of iCC (bile ductular adenocarcinoma) as yet in Japanese literature $(4,12,16)$. By others, as in the latest edition [2010] of WHO tumor classification, and because it is thought to originate from HPCs located in ductules/ canals of Hering $(37,39)$, it is classified as a subtype of combined hepatocellular-cholangiocarcinoma. This concept is actually revisited and the achievement of a consensus terminology is needed in the coming years. Immunohistochemical profile of CLC seems rather in favour of a biphenotypic (hepatobiliary) primary liver carcinoma. Indeed, CLC shares iCC immunomarkers, such as CK7 and CK19, but also HPCs marker (N-CAM) and hepatocytic differentiation marker (HepPar1), helpful for distinguishing CLC from classical well-differentiated iCC $(12,16,37,38)$. First molecular studies on CLCs seem to show surprisingly more homogeneity than the immunohistochemical phenotype, with a profile that is closer to iCC than HCC (40). In view of these aspects, CLCs could be include in a category of tumors in-between conventional combined HCC-CC and iCC: the category of pure iCC with stem cell features and hepatocytic markers expression in immunohistochemistry (38).

\section{Differential diagnosis of iCC}

\section{Combined hepatocellular-cholangiocarcinoma}

Combined hepatocellular cholangiocarcinoma (CHC) is a rare tumor representing $0.4-14.2 \%$ of primary liver cancer and is characterized by pathological features of both HCC and cholangiocarcinoma $(1,41,42)$. Macroscopically, combined HCC-CC may mimic iCC (Figure 7). Since this entity was first reported in 1949 , the classification of $\mathrm{CHC}$ has constantly evolved. The latest classification by the $\mathrm{WHO}$ relied on the hypothesis that $\mathrm{CHC}$ arises from hepatic progenitor cells. Yet, there is no evidence demonstrating the common origin of different components of CHC. In addition, the definition of CHC subtypes is still ambiguous and the identification of CHC subtype when a single tumor contains several components is still unresolved. Finally, the role that plays the different $\mathrm{CHC}$ components for prognosis and outcome of this disease is not clear. Currently, according to the WHO (1), CHC is classifed into the classical subtype and subtype with stem-cell features. The latest 2010 WHO classification recognized combined hepatocellular-cholangicarcinoma as a mixed tumor with unequivocal and closely intermingled areas of both HCC and cholangiocarcinoma. This tumor is to distinguish from collision tumor where both independent HCC and CC coexist within the same liver (either at distance or as adjacent tumors).

\section{HCC}

Architecturally, while iCC may have trabecular pattern, HCC may be pseudoglandular, potentially complicating their histological distinction. However unlike iCC, HCC does not display true glands or mucin, while it 

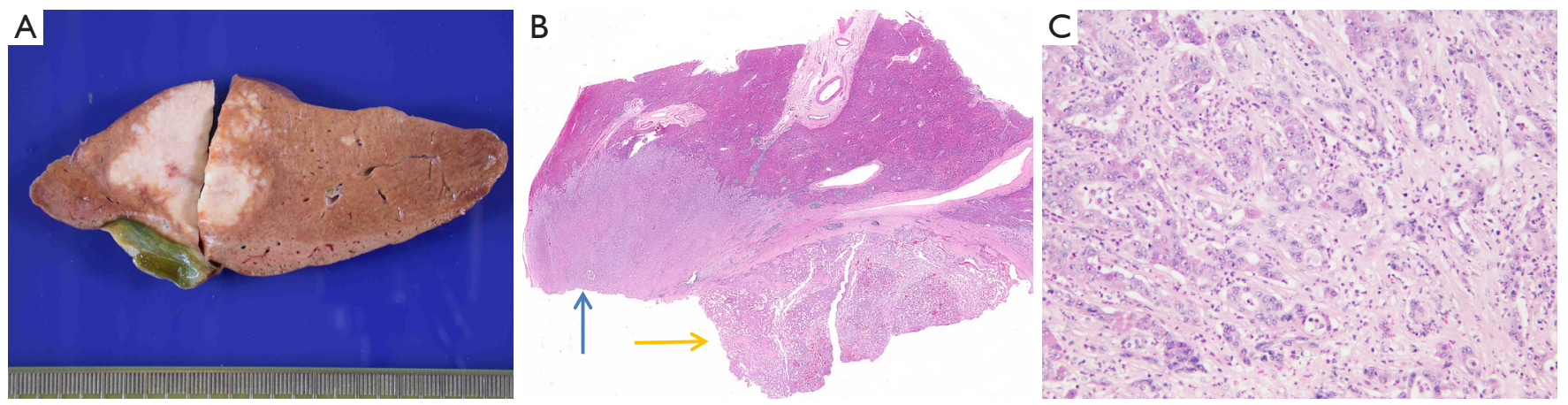

Figure 7 Conventional combined hepatocellular-cholangiocarcinoma. (A) Gross specimen showing a unique whitish lesion, relatively well demarcated, similar to a classical cholangiocarcinoma; (B) hematoxylin and eosin stain. Whole section demonstrating a tumor composed of two distinct components: a cholangiocarcinoma component (blue arrow) and a hepatocellular carcinoma component (yellow arrow); (C) hematoxylin and eosin stain. Intermediate area with a gradual transition between the two components : hepatocellular carcinoma component in the upper left corner and cholangiocarcinoma component in the lower right corner, separated by pseudoacinar formations lined not by hepatocytes, but by cuboidal, cholangiocyte-like cells (original magnification, $\times 20$ ).

produces bile, and has habitually prominent nucleoli. Differential diagnosis may be challenging in the case of a fibrotic stoma rich variant of HCC (see below). Immunohistochemically, iCC usually does not express specific hepatocytic markers as AFP, glypican-3, CK8 and 18, BSEP or MDR3 (commonly expressed by HCC) with a few exceptions for HepPar1 and arginase 1 (20), but may rather frequently express, at a mild level, albumin mRNA, while, unlike HCC, it shows diffuse cytoplasmic labeling with polyclonal CEA, monoclonal CEA and CA199. A focal expression of CK7 and of CK19 is detected in $15 \%$ and $10 \%$ to $27 \%$ of HCC, respectively. HCC expressing HPC or ductular markers like CK19 emphasis a subcategory of HCC with stemness features and display a more aggressive clinical course. CK19 positivity in HCC is a significant predictor of worse overall survival and of early postoperative recurrence in these patients.

\section{Scirrbous HCC}

HCC with large zone ( $>50 \%$ of area) of fibrous stroma (scirrhous pattern) may possibly be misdiagnosed as iCC both at imaging as at gross assessment (43). Macroscopically it is frequently characterized by a white-grayish, fibrotic, solid, well demarcated but not encapsulated subcapsular mass. At microscopic level, schirrous HCC shows diffuse bands of fibrosis along sinusoid-like blood spaces intermingled with tumoral cell trabeculae of variable width. Key feature for diagnosis is tumor cell morphology analogous to regular HCC (Figure 8).
Immunohistochemistry investigations have pointed up a considerable high expression of cytokeratin 7 ( $>60 \%$ of cases) and a significantly lower expression of HepPar1 in scirrhous HCC than in ordinary HCC, underlining a peculiar histogenesis.

\section{Metastase}

Because of lack of an entirely specific marker, diagnosis of iCC is established by exclusion of metastatic adenocarcinoma. Basic immunohistochemical panel combining CK7, CK20, CDX-2, TTF-1, Estrogen and Progesteron receptors, BRST-2 and PSA should be chosen according to the clinical setting, contributing to exclude hepatic metastases form common primary sites including colon, lung, breast and prostate. iCC is typically diffusely positive for CK7, and negative or only slightly positive for CK20 and other previously cited markers. Metastatic carcinoma from gallbladder, pancreas or upper gastrointestinal tract can't be easily distinguished neither morphologically nor by immunostainings from iCC. Albumin mRNA ISH is a sensitive and highly specific diagnostic tool for distinguishing iCC from metastatic adenocarcinoma, particularly pancreatic ductal adenocarcinoma (21).

\section{Bile duct adenoma (BDA)}

It may occur as an incidental finding, possibly confounded with iCC. It was initially considered as a PBG hamartoma 

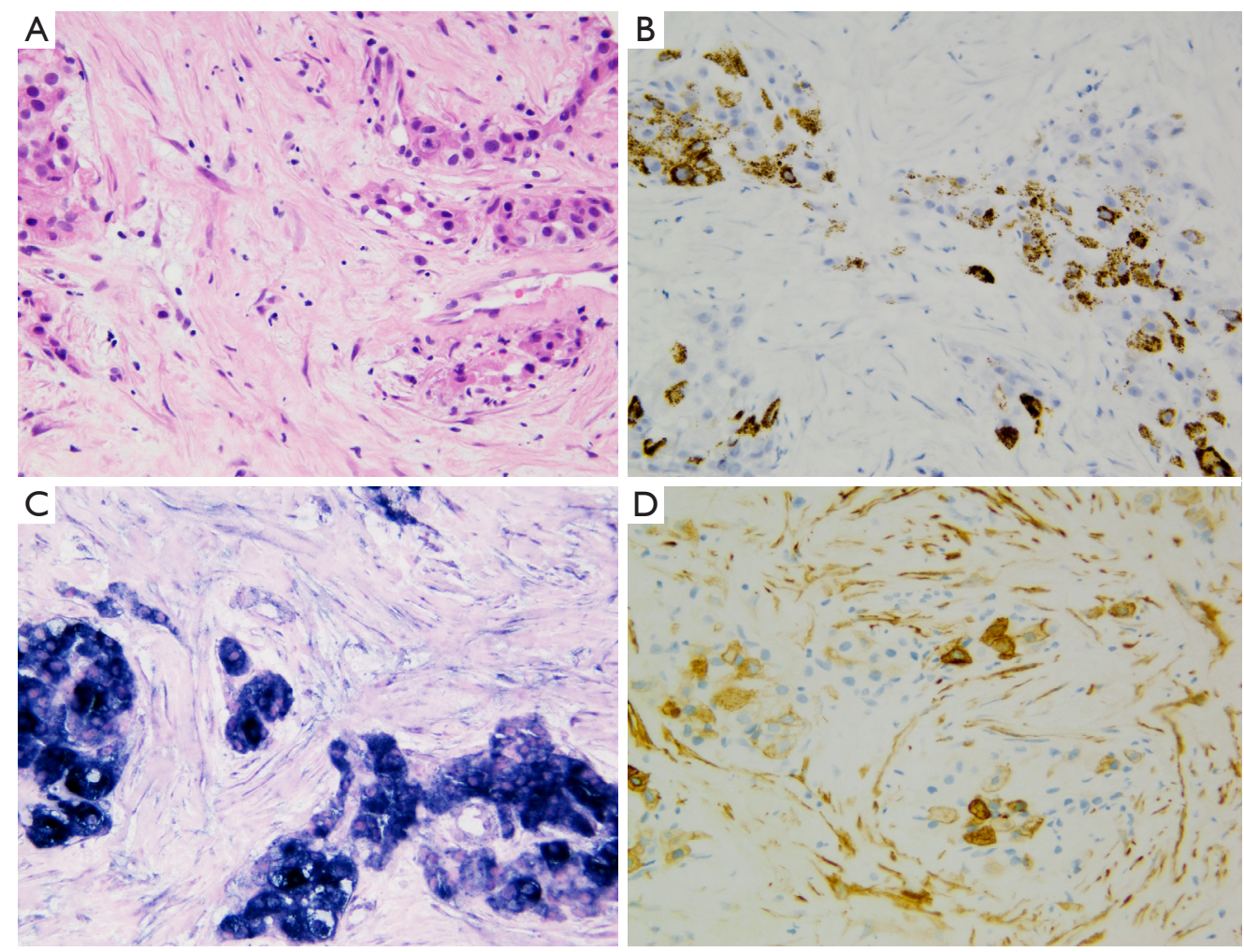

Figure 8 Scirrhous hepatocellular carcinoma. (A) Hematoxylin and eosin stain. The carcinoma shows a hepatoid morphology with polygonal tumor cells containing moderate eosinophilic cytoplasm, invading an abundant fibrous stroma, without any glandular differentiation or mucin production (original magnification, $\times 40$ ); (B) HepPar1 stain. Tumor cells are characterized by a lower expression of HepPar1 in a portion of cells, in comparison with a classical hepatocellular carcinoma (original magnification, $\times 40$ ); (C) albumin mRNA in situ hybridization (ISH). All of the tumor cells demonstrate an intense cytoplasmic hybridization signal (original magnification, $x 40$ ); (D) cytokeratin 7 stain. Tumor cells show a higher expression of cytokeratin 7 in comparison with a classical hepatocellular carcinoma (original magnification, $\times 40)$.

or a localized reactive ductular proliferation in response to a previous unknown injury. The detection of BRAF V600E mutation in a significant number of BDAs in a study by Pujals (44) has led to consider BDA as a true benign neoplasm. It is typically subcapsular, measuring from 1 to $20 \mathrm{~mm}$, and is macroscopically firm, gray-white, tan or yellow, round and well circumscribed but not encapsulated, most often forming a solitary mass. Histologically, benign, noncystic ductules and variable degrees of inflammation and fibrosis characterize BDA (Figure 9). The immunophenotype of these ductules was similar to that of interlobular BDs, notably with albumin expression reported in $92 \%$ of cases (45). The absence of bile and cystic changes and lack of association with polycystic disease of the liver and kidneys are the main features distinguishing BDA from von Meyenburg complex.

\section{Conclusions}

Because of increasing incidence of iCC [notably in nonendemic areas of parasitic biliary infection and especially in relation to non-biliary chronic liver disease as chronic hepatitis $\mathrm{C}$ virus (HCV) infection], this cancer is today a matter of intense research and reconsideration. On the basis of anatomical location, cholangiocarcinomas were till recently essentially classified into intrahepatic CCs, perihilar CCs and distal CCs. Taking into account its considerable morphological heterogeneity and most likely different pathogenic mechanisms and several unique biological characteristics, several classifications have been proposed. Notably, iCC includes several macroscopic and histologic conventional and unconventional subtypes. Knowledge about iCC histogenesis is thus in broadening. 

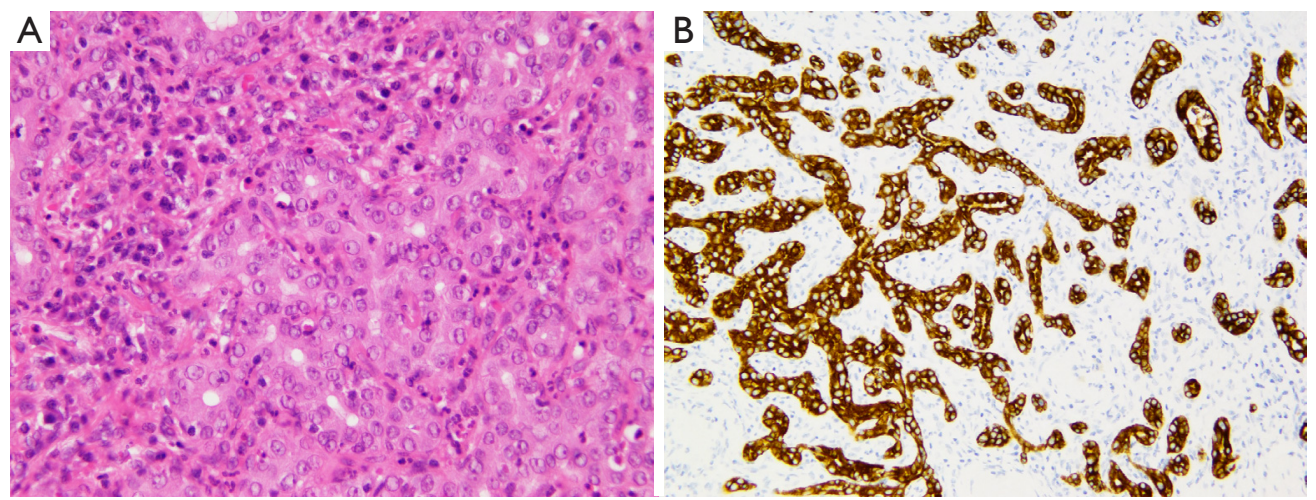

Figure 9 Bile duct adenoma. (A) Hematoxylin and eosin stain. Benign tumor represented by small tubular structures lined by a single layer of non-atypical cubocylindrical cells admixed with variable inflammation and fibrosis (original magnification, $\times 40$ ); (B) cytokeratin 7 stain. All tubular structures are well demarcated with the cytokeratin 7 stain (original magnification, $\times 20$ ).

Latest (mostly experimental) data put forwards that iCC may have multiple cellular origins, including not only intrahepatic cholangiocytes but also differentiated hepatocytes, pluripotent stem cells [such as hepatic (HPCs) and biliary tree (BTSCs) stem/progenitor cells] and PBG.

\section{Acknowledgements}

None.

\section{Footnote}

Conflicts of Interest: The authors have no conflicts of interest to declare.

\section{References}

1. Bosman FT, Carneiro F, Hruban RH, et al. WHO Classification of Tumours of the Digestive System, Fourth Edition. WHO Classification of Tumours, IARC WHO Classification of Tumours, No 3, 2010.

2. Brierley JD, Gospodarowicz MK, Wittekind C. editors. TNM Classification of Malignant Tumours, Seventh Edition. Wiley: Wiley-Blackwell, 2009.

3. Mansour JC, Aloia TA, Crane CH, et al. Hilar cholangiocarcinoma: expert consensus statement. HPB (Oxford) 2015;17:691-9.

4. Nakanuma Y, Miyata T, Uchida T. Latest advances in the pathological understanding of cholangiocarcinomas. Expert Rev Gastroenterol Hepatol 2016;10:113-27.

5. Sia D, Tovar V, Moeini A, et al. Intrahepatic cholangiocarcinoma: pathogenesis and rationale for molecular therapies. Oncogene 2013;32:4861-70.

6. Cardinale V, Bragazzi MC, Carpino G, et al. Cholangiocarcinoma: increasing burden of classifications. Hepatobiliary Surg Nutr 2013;2:272-80.

7. Sakamoto Y, Kokudo N, Matsuyama Y, et al. Proposal of a new staging system for intrahepatic cholangiocarcinoma: Analysis of surgical patients from a nationwide survey of the Liver Cancer Study Group of Japan. Cancer 2016;122:61-70.

8. Nakajima T, Kondo Y, Miyazaki M, et al. A histopathologic study of 102 cases of intrahepatic cholangiocarcinoma: histologic classification and modes of spreading. Hum Pathol 1988;19:1228-34.

9. Sano T, Kamiya J, Nagino M, et al. Macroscopic classification and preoperative diagnosis of intrahepatic cholangiocarcinoma in Japan. J Hepatobiliary Pancreat Surg 1999;6:101-7.

10. Yamasaki S. Intrahepatic cholangiocarcinoma: macroscopic type and stage classification. J Hepatobiliary Pancreat Surg 2003;10:288-91.

11. Sanada Y, Kawashita Y, Okada S, et al. Review to better understand the macroscopic subtypes and histogenesis of intrahepatic cholangiocarcinoma. World J Gastrointest Pathophysiol 2014;5:188-99.

12. Sempoux C, Jibara G, Ward SC, et al. Intrahepatic cholangiocarcinoma: new insights in pathology. Semin Liver Dis 2011;31:49-60.

13. Yamamoto $M$, Takasaki K, Yoshikawa T. Lymph node metastasis in intrahepatic cholangiocarcinoma. Jpn J Clin Oncol 1999;29:147-50.

14. Imai K, Yamamoto M, Ariizumi S. Surgery for periductal infiltrating type intrahepatic cholangiocarcinoma without 
hilar invasion provides a better outcome than for massforming type intrahepatic cholangiocarcinoma without hilar invasion. Hepatogastroenterology 2010;57:1333-6.

15. Komuta M, Govaere O, Vandecaveye V, et al. Histological diversity in cholangiocellular carcinoma reflects the different cholangiocyte phenotypes. Hepatology 2012;55:1876-88.

16. Sempoux C, Fan C, Singh P, et al. Cholangiolocellular carcinoma: an innocent-looking malignant liver tumor mimicking ductular reaction. Semin Liver Dis 2011;31:104-10

17. Nakanuma Y, Sato Y, Ikeda H, et al. Intrahepatic cholangiocarcinoma with predominant "ductal plate malformation" pattern: a new subtype. Am J Surg Pathol 2012;36:1629-35.

18. Sato Y, Harada K, Sasaki M, et al. Clinicopathological significance of $\mathrm{S} 100$ protein expression in cholangiocarcinoma. J Gastroenterol Hepatol 2013;28:1422-9.

19. Aishima S, Fujita N, Mano Y, et al. Different roles of S100P overexpression in intrahepatic cholangiocarcinoma: carcinogenesis of perihilar type and aggressive behavior of peripheral type. Am J Surg Pathol 2011;35:590-8.

20. Fujikura K, Yamasaki T, Otani K, et al. BSEP and MDR3: Useful Immunohistochemical Markers to Discriminate Hepatocellular Carcinomas From Intrahepatic Cholangiocarcinomas and Hepatoid Carcinomas. Am J Surg Pathol 2016;40:689-96.

21. Ferrone CR, Ting DT, Shahid M, et al. The Ability to Diagnose Intrahepatic Cholangiocarcinoma Definitively Using Novel Branched DNA-Enhanced Albumin RNA In Situ Hybridization Technology. Ann Surg Oncol 2016;23:290-6.

22. Nakanuma Y, Tsutsui A, Ren XS, et al. What are the precursor and early lesions of peripheral intrahepatic cholangiocarcinoma? Int J Hepatol 2014;2014:805973.

23. Sato Y, Harada K, Sasaki M, et al. Histological Characterization of Biliary Intraepithelial Neoplasia with respect to Pancreatic Intraepithelial Neoplasia. Int J Hepatol 2014;2014:678260.

24. Sato Y, Harada K, Sasaki M, et al. Cystic and micropapillary epithelial changes of peribiliary glands might represent a precursor lesion of biliary epithelial neoplasms. Virchows Arch 2014;464:157-63.

25. Aishima S, Kubo Y, Tanaka Y, et al. Histological features of precancerous and early cancerous lesions of biliary tract carcinoma. J Hepatobiliary Pancreat Sci 2014;21:448-52.

26. Onoe S, Shimoyama Y, Ebata T, et al. Prognostic delineation of papillary cholangiocarcinoma based on the invasive proportion: a single-institution study with 184 patients. Surgery 2014;155:280-91.

27. Rougemont AL, Genevay M, McKee TA, et al. Extensive biliary intraepithelial neoplasia (BilIN) and multifocal early intrahepatic cholangiocarcinoma in non-biliary cirrhosis. Virchows Arch 2010;456:711-7.

28. Wu TT, Levy M, Correa AM, et al. Biliary intraepithelial neoplasia in patients without chronic biliary disease: analysis of liver explants with alcoholic cirrhosis, hepatitis $\mathrm{C}$ infection, and noncirrhotic liver diseases. Cancer 2009;115:4564-75.

29. Zen Y, Fujii T, Itatsu K, et al. Biliary papillary tumors share pathological features with intraductal papillary mucinous neoplasm of the pancreas. Hepatology 2006;44:1333-43.

30. Zen Y, Fujii T, Itatsu K, et al. Biliary cystic tumors with bile duct communication: a cystic variant of intraductal papillary neoplasm of the bile duct. Mod Pathol 2006;19:1243-54.

31. Zen Y, Aishima S, Ajioka Y, et al. Proposal of histological criteria for intraepithelial atypical/proliferative biliary epithelial lesions of the bile duct in hepatolithiasis with respect to cholangiocarcinoma: preliminary report based on interobserver agreement. Pathol Int 2005;55:180-8.

32. Oikawa T. Cancer Stem cells and their cellular origins in primary liver and biliary tract cancers. Hepatology 2016;64:645-51.

33. Wei M, Lü L, Lin P, et al. Multiple cellular origins and molecular evolution of intrahepatic cholangiocarcinoma. Cancer Lett 2016;379:253-61.

34. Kok CY, Miyajima A, Itoh T. Adaptive remodeling of the biliary tree: the essence of liver progenitor cell expansion. J Hepatobiliary Pancreat Sci 2015;22:546-50.

35. Strazzabosco M, Fabris L. Functional anatomy of normal bile ducts. Anat Rec (Hoboken) 2008;291:653-60.

36. Strazzabosco M, Spirlí C, Okolicsanyi L. Pathophysiology of the intrahepatic biliary epithelium. J Gastroenterol Hepatol 2000;15:244-53.

37. Komuta M, Spee B, Vander Borght S, et al. Clinicopathological study on cholangiolocellular carcinoma suggesting hepatic progenitor cell origin. Hepatology 2008;47:1544-56.

38. Brunt EM, Paradis V, Sempoux C, et al. Biphenotypic (hepatobiliary) primary liver carcinomas: the work in progress. Hepat Oncol 2016;2:255-73.

39. Woo HG, Lee JH, Yoon JH, et al. Identification of a cholangiocarcinoma-like gene expression trait in 
hepatocellular carcinoma. Cancer Res 2010;70:3034-41.

40. Coulouarn C, Cavard C, Rubbia-Brandt L, et al.

Combined hepatocellular-cholangiocarcinomas exhibit progenitor features and activation of $\mathrm{Wnt}$ and TGF $\beta$ signaling pathways. Carcinogenesis 2012;33:1791-6.

41. Kim SH, Park YN, Lim JH, et al. Characteristics of combined hepatocelluar-cholangiocarcinoma and comparison with intrahepatic cholangiocarcinoma. Eur J Surg Oncol 2014;40:976-81.

42. Cazals-Hatem D, Rebouissou S, Bioulac-Sage P, et al. Clinical and molecular analysis of combined hepatocellular- cholangiocarcinomas. J Hepatol 2004;41:292-8.

43. Matsuura S, Aishima S, Taguchi K, et al. 'Scirrhous' type hepatocellular carcinomas: a special reference to expression of cytokeratin 7 and hepatocyte paraffin 1. Histopathology 2005;47:382-90.

44. Pujals A, Bioulac-Sage P, Castain C, et al. BRAF V600E mutational status in bile duct adenomas and hamartomas. Histopathology 2015;67:562-7.

45. Moy AP, Arora K, Deshpande V. Albumin expression distinguishes bile duct adenomas from metastatic adenocarcinoma. Histopathology 2016;69:423-30.

Cite this article as: Vijgen S, Terris B, Rubbia-Brandt L. Pathology of intrahepatic cholangiocarcinoma. HepatoBiliary Surg Nutr 2017;6(1):22-34. doi: 10.21037/hbsn.2016.11.04 